\title{
Expression of PI3K/AKT Signaling Pathway in IFN- $\beta$-induced Differentiation of Human Mesenchymal Stem Cells into Chondroblasts
}

\author{
Zhang Li-Jun ${ }^{1}$, Huang Zhi-Li ${ }^{1}$, Wang Yan ${ }^{1}$, Li Qiao-Qiao ${ }^{1}$
}

\author{
${ }^{1}$ School of Applied Chemisty and Biotechnology, Shenzhen Polytechnic, Shenzhen, Guangdong, China
}

\begin{abstract}
Induction of chondrogenic differentiation from MSCs are necessary for qualification as sources of seed cells for cartilage tissue engineering o Our previous research showed IFN- $\beta$ maybe as a helper of TGF- $\beta$ to promote chondrogenesis of hMSCs. In this paper, we investigated the expression of PI3K/AKT during the chondrogenic differentiation of hMSCs, hMSCs were divided into Blank control group, TGF- $\beta 3$ group and TGF- $\beta 3+$ IFN- $\beta 1$ a group and then were induced into cartilage pellets respectively. At day 4,7,14,21, the pellets were collected and qRT-PCR and Western blotting were used to detect the relative expression of PI3K and AKT. The qPCR results showed that the mRNA expression levels of PI3K and AKT were up-regulated by TGF- $\beta 3$ induced. Furthermore, the synergetic effect of TGF- $\beta 3$ and IFN- $\beta 1$ a would up-regulate the mRNA expression levels of PI3K and AKT significantly. The Western blotting results showed that TGF- $\beta 3$ also could up-regulate the expression of PI3K protein and with IFN- $\beta 1$ a the expression level increased significantly. However, according to AKT protein, TGF- $\beta 3$ maybe only could up-regulate AKT(S473) expression, and with IFN- $\beta 1$ a the AKT(T308) expression could be up-regulated .These results showed that the synergetic effect of TGF- $\beta 3$ and IFN- $\beta 1$ a could up-regulate the gene expression in PI3K/AKT signaling pathway and then promote hMSCs chondrogenic differentiation.
\end{abstract}

Keywords: PI3K/AKT Signaling Pathway, hMSCs, Chondrogenic Differentiation

\section{Introduction}

Over the past few years, progress has been made in the study on osteogenesis of mesenchymal stem cells (MSCs) and regulation of chondrogenic differentiation $[1,2]$. Directed induction of osteogenesis and chondrogenic differentiation from MSCs are necessary for qualification as sources of seed cells for bone and cartilage tissue engineering [3,4]. This area has become a focus in regenerative medicine, tissue engineering, and wound healing research.

Osteogenesis and chondrogenic differentiation depend heavily on cytokines and growth factors, and a number of such factors have been studied in the field of cartilage tissue engineering. These include transforming growth factors 1 and 3 (TGF- $\beta 1$ and TGF- $\beta 3$ ),morphogenic proteins (BMP), fibroblast growth factor 2 (FGF-2), Insulin-like growth factor I (IGF-I), vascular endothelial growth factor(VFGF),Epidermal growth factor (EGF), hepatocyte growth factor (HGF), platelet-derived growth factor (PDGF) [5-10]。 These factors first bind to their cognate receptors on the stem cell membrane and thus trigger the cellular signaling pathways such as Wnt/ $\beta$-catenin, MAPK/ERK and Notch, resulting in activation of downstream signaling molecules and regulate expression of the cartilage-related genes
Sox-9,RUNX-2,Oct-4,Nanog, ultimately affecting capability of chondrogenic differentiation[11-15]. With the recent increases in our knowledge regarding the actions of various growth factors, some less important growth factors have been ruled out and more interactions among growth factors continue to be discovered. We have previously reported that combination of recombinant IFN- $\beta$ and TGF- $\beta$ significantly can promote differentiation of MSCs into chondrocyte. However, the mechanisms underlying its effects are currently under investigation [16].

PI3K-Akt Pathway is an important intracellular signal transduction pathway contributes cell functions such as growth, proliferation, differentiation, metabolism, cytoskeletal arrangement, protein synthesis and degradation, apoptosis, and the cell cycle. The Key proteins involved in this pathway are phosphatidylinositol 3-kinase (PI3K) and Akt/Protein Kinase B. Growth factors can bind to receptor tyrosine kinase (RTKs) and activate PI3K, which phosphorylates phosphatidylinositol-4,5-bisphosphate (PIP2) to generate phosphatidylinositol-3,4,5-bisphosphate (PIP3). PIP3 bind to proteins AKT and PDK1, then PDK1 phosphorylates AKT at T308 to activate AKT. AKT

This article is published under the terms of the Creative Commons Attribution License 4.0 Author(s) retain the copyright of this article. Publication rights with Alkhaer Publications.

Published at: http://www.ijsciences.com/pub/issue/2019-08/

DOI: 10.18483/ijSci.2156; Online ISSN: 2305-3925; Print ISSN: 2410-4477 
can phosphate transcription factors to promote cell proliferation and growth. [17-19]

In this paper, we investigated the expression of PI3K and Akt during hMSCs chondrogenic differentiation along with treatment of IFN- $\beta$ to verify if the IFN- $\beta$ mediate PI3K/AKT signaling pathway to promote hMSCs chondrogenic differentiation.

\section{Methods}

\section{1 hMSCs culture}

hMSCs (provided by Cyagen Bioscience Inc.,Guangzhou, China) were seeded in $25 \mathrm{ml}$ culture flask in low glucose DMEM (Gibco, Grand Island, SÁ) containing $10 \%$ fetal bovine serum (Gibco), $100 \mathrm{U} / \mathrm{mL}$ penicillin, and $100 \mu \mathrm{g} / \mathrm{mL}$ streptomycin (Gibco). Passaged the cells when they grow to $80 \%$ according to the conventional methods.

\subsection{Induction of chondrogenic differentiation from hMSCs with IFN- $\beta$}

hMSCs at passage 5 were collected and divided to three groups: control, TGF- $\beta 3$, and TGF- $\beta 3+$ IFN- $\beta 1 \mathrm{a}$. The compositions of these culture groups are as follows: 1) control medium: basal chondrogenic medium [high glucose DMEM containing $100 \mathrm{U} / \mathrm{mL}$ penicillin and $100 \mu \mathrm{g} / \mathrm{mL}$ streptomycin, 100nM dexamethasone, 50 $\mathrm{mg} / \mathrm{ml}$ ascorbic acid-2-phosphate, $100 \mathrm{mg} / \mathrm{ml}$ sodium pyruvate, $40 \mathrm{mg} / \mathrm{ml}$ proline and $1 \%$ insulin, transferring sodium selenite mix] (Cyagen Bioscience Inc); 2) TGF- $\beta 3$ group medium: control medium +10 ng/ml TGF- $\beta 3$; 3 ) TGF- $\beta 3+$ IFN- $\beta 1$ a group medium: control medium $+10 \mathrm{ng} / \mathrm{ml}$ TGF- $\beta 3+100 \mathrm{ng} / \mathrm{ml}$ IFN- $\beta 1$. All of the cell groups were cultured in $15 \mathrm{ml}$ centrifuge tubes with loose caps (for air exchange) were cultured in a $\mathrm{CO}_{2}$ incubator $\left(37^{\circ} \mathrm{C}, 5 \% \mathrm{CO}_{2}\right)$. After $24 \mathrm{~h}$ of incubation, the tubes were flicked at bottom to loosen the cartilage pellet until it became suspended in the culture medium. Culture medium was changed every 3 days in this manner.

\subsection{RNA extraction and quantitative RT-PCR analysis}

At days 4, 7, 14, and 21 of chondrogenic differentiation, cell pellets from each group were harvested for qRT-PCR analysis. Total RNA was acquired using the RNeasy Mini Kit (Qiagen, Hilden, Germany) and reverse transcribed using a SuperScript III First-Strand Synthesis System Kit (Invitrogen, Carlsbad, SÁ). Primers are listed in Table 1 and iQ SYBR Green Supermix (Bio-Rad, Hercules, CA) was performed for qRT-PCR analysis. Relative expression of each target mRNA was calculated by $2^{-\Delta \Delta \mathrm{Ct}}$ method.

Table 1 Sequences of the primers for real-time PCR

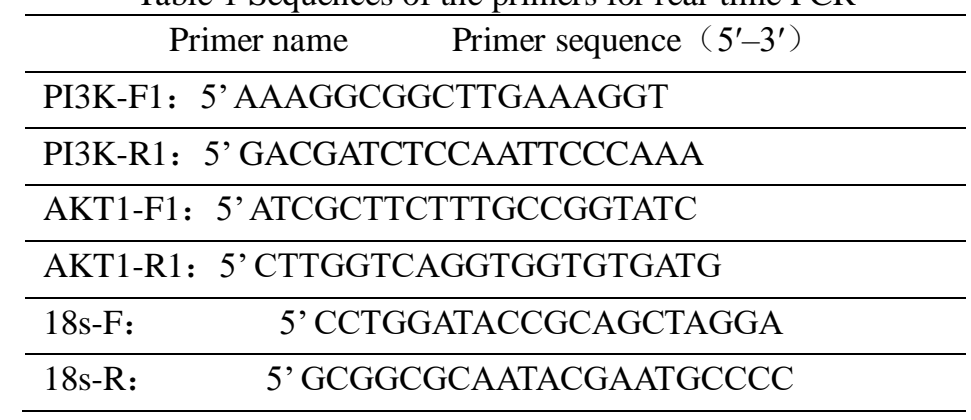

\subsection{Protein extraction and Western blot}

At days 4, 7, 14, and 21, Total protein from each chondrogenic differentiation group were extracted respectively for Western blot. The protein concentration was detected using BCA kit (Thermo Fisher Scientific). Approximately 10-30 $\mu \mathrm{g}$ protein samples/well run on SDS-PAGE and transferred to an Immobilon-P membrane (Millipore Corp., Bedford, MA). Membranes were probed with primary antibodies: anti-PI3K (Abcam, Cambridge, UK), anti-AKT(T308) and anti-AKT(S473) (Abcam, Cambridge, UK). Anti-GAPDH (Abcam) as reference. Immunoblotted bands were visualized using ECL reagents (GE, Buckinghamshire, UK). The signal intensity of each target protein was quantified using densitometry and normalized to that of GAPDH.

\subsection{Statistics}

Parametric data are presented as means \pm SD from three replicate samples. Difference compared using two-way ANOVA or a Student's t-test $(p<0.05$ was considered statistically significant).

\section{Results}

\subsection{PI3K mRNA expression in hMSCs during} chondrogenic differentiation

$\mathrm{PI} 3 \mathrm{~K}$ is a key gene in PI3K/AKT signaling pathway. Here, we first assessed PI3K mRNA expression in hMSCs during chondrogenic differentiation. As shown in Figure 1, at the 4 points in time post-induction, PI3K expression was higher in the TGF- $\beta 3$ group than in the control group $(P<0.05)$, and peak expression was observed at day 21 after induction. These results indicate that the PI3K/AKY signaling pathway is activated during chondrogenic differentiation of hMSCs. In comparison, PI3K expression at all 4 points in time was the highest in the TGF- $\beta 3+$ IFN- $\beta 1$ a group, higher than both the control group $(P<0.01)$ and 
TGF- $\beta 3$ group. We also found PI3K expression to be upregulated as induction time went on, so that on day 21 post-induction, PI3K expression in TGF- $\beta 3+$ IFN- $\beta 1$ a group was twice that in the TGF- $\beta 3$ group.
These results suggest that during chondrogenic differentiation from hMSCs, TGF- $\beta 3$ may activate the $\mathrm{PI} 3 \mathrm{~K} / \mathrm{AKT}$ signaling pathway. This effect of TGF- $\beta 3$ may be rendered more intense by addition of IFN- $\beta 1 \mathrm{a}$.

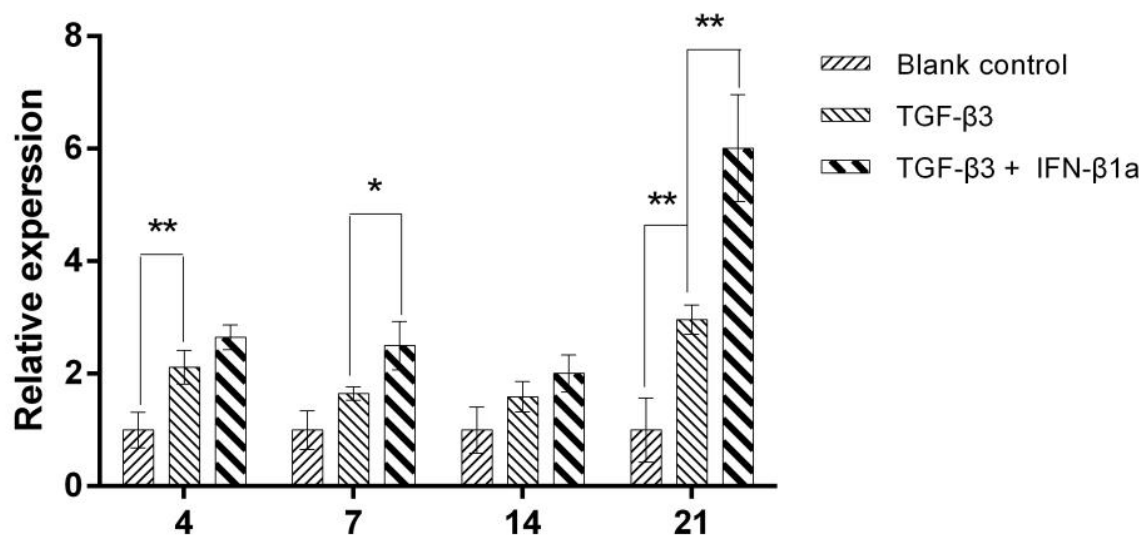

Culture time (d)

Fig.1 qRT-PCR result of PI3K gene during the chondrogenic differentiation of hMSCs $* * \mathrm{P}<0.01,{ }^{*} \mathrm{P}<0.05$

3.2. AKT mRNA expression in hMSCs during chondrogenic differentiation

AKT is a gene downstream of PI3K in the PI3K/AKT signaling pathway. For this reason, after assessing PI3K expression during chondrogenic differentiation of hMSCs, we further examined AKT mRNA expression. As shown in Figure 2, at 4 points in time post-induction, AKT mRNA expression was higher in both the TGF- $\beta 3$ and TGF- $\beta 3+$ IFN- $\beta 1$ a groups than in the control group $(P<0.05)$. AKT expression levels increased over time. AKT expression in both treatment groups was significantly higher than in the control group on day 7 post-induction $(P<0.01)$, and this difference was greater on day 14 post-induction. These results further indicate that, during chondrogenic differentiation of hMSCs, PI3K gene activation may cause further activation of its downstream AKT gene after induction. TGF- $\beta 3+$ IFN- $\beta 1$ la treatment induced higher AKT expression than TGF- $\beta 2$ alone, especially at 21 days post-induction $(P<0.01)$. These results also indicate that IFN- $\beta 1$ a may amplify the effect of TGF- $\beta 3$ in promoting PI3K/AKT signaling pathway.



Fig. 2 qRT-PCR result of AKT gene during the chondrogenic differentiation of hMSCs. $* * \mathrm{P}<0.01, * \mathrm{P}<0.05$

3.3. Expression of key gene products in hMSCs during chondrogenic differentiation

After obtaining the results of two key genes in the
PI3K/AKT signaling pathway, PI3K and AKT, we used Western blot to determine the concentrations of protein products in these two genes. As shown in 
Figure $3 \mathrm{~B}$, at all points in time except for day 7 post-induction, PI3K protein expression was higher in the TGF- $\beta 3$ group than in the control group $(P<0.05)$. In the TGF- $\beta 3+$ IFN- $\beta 1$ a group, however, PI3K protein expression was higher than in the control group at all 4 points in time post-induction $(P<0.01)$. At some points in time, it was also higher than in the TGF- $\beta 3$ group. $\mathrm{PI} 3 \mathrm{~K}$ protein expression increased over time and peaked on day 21 post-induction for TGF- $\beta 3+$ IFN- $\beta 1$ a group, which was almost twice that in the TGF- $\beta 3$ group $(1.89 \times, P<0.01)$, which was similar to the observed in mRNA expression level.

Because activation of AKT protein is characterized by phosphorylation at Ser473 and Thr308, we determined protein expression levels of AKT(T308) and AKT(S473). The intensity of expression of these two proteins is shown in Figure $3 \mathrm{C}$ and $\mathrm{D}$, respectively. $\mathrm{AKT}(\mathrm{S} 473)$ expression during induction was higher in

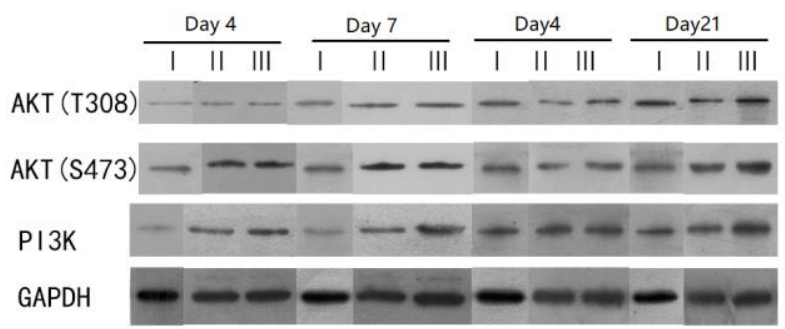

(A)
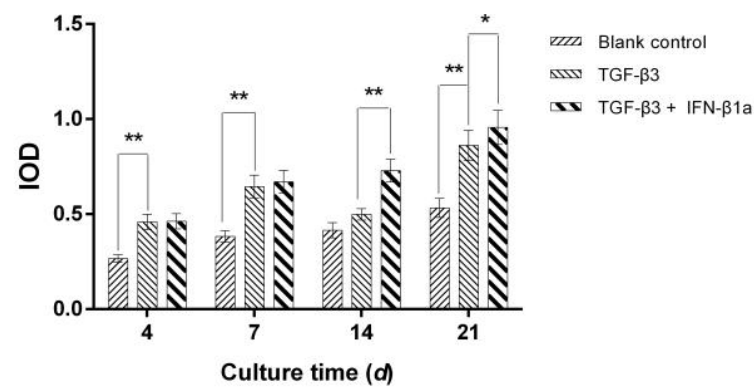

(C) both TGF- $\beta 3$ and TGF- $\beta 3+$ IFN- $\beta 1$ a groups compared to the control group $(P<0.05)$, the expression levels increased over time. When compared to the TGF- $\beta 3+$ IFN- $\beta 1$ a group had higher AKT(S473) expression on day 14 post-induction than the TGF- $\beta 3$ group $(P<0.01)$. AKT(T308) expression was higher in the TGF- $\beta 3+$ IFN- $\beta 1$ a group than in either the control or TGF- $\beta 3$ groups on day 14 post-induction. On day 21 post-induction, AKT(T308) protein expression in TGF- $\beta 3+$ IFN- $\beta 1$ a group was twice that in the TGF- $\beta 3$ group $(2.03 \times, P<0.01)$. These results indicate that TGF- $\beta 3$ mainly upregulates AKT(S473) expression while IFN- $\beta 1$ a may work synergistically with TGF- $\beta 3$ in promoting $\mathrm{AKT}(\mathrm{T} 308)$ protein expression. The results of PI3K, AKT(S473), and AKT(T308) protein expression during induction of hMSC differentiation into chondroblasts indicate that IFN- $\beta 1$ a may work together with TGF- $\beta 3$ to activate the PI3K/AKT signaling pathway.

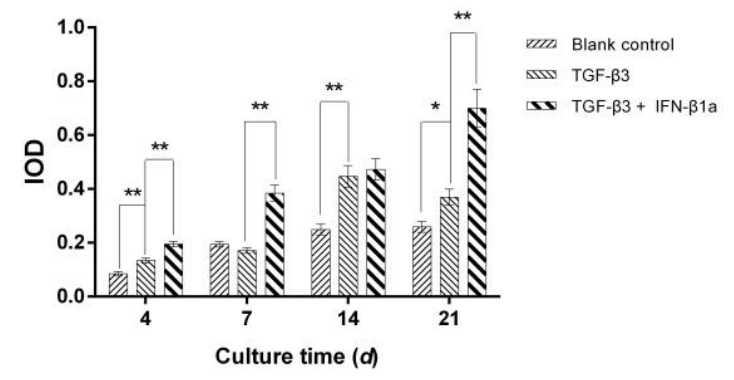

(B)

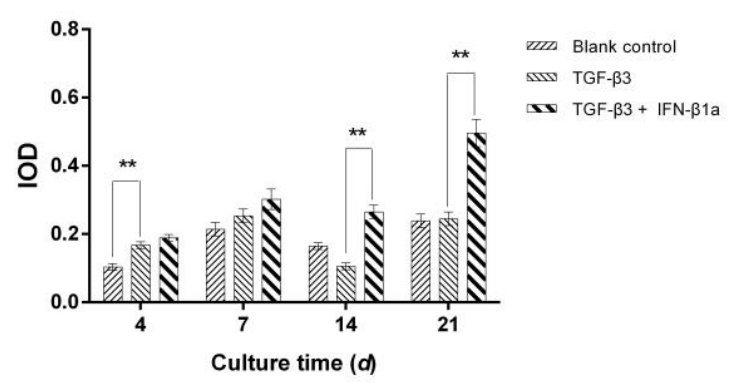

(D)

Fig. 4-2 Western blotting result of PI3K and AKT in hMSCs.

A: Western blot results; B: IOD analysis of PI3K; C: IOD analysis of AKT(S473) ;(D): IOD analysis of (T308); I: Control group; II: TGF- $\beta 3$ group; III: TGF- $\beta 3+$ IFN- $\beta 1$ a group

$* * \mathrm{P}<0.01, * \mathrm{P}<0.05$

\section{Discussion}

Recent studies have shown the PI3K-AKT signaling pathway to be closely related to proliferation and differentiation of stem cells. The specific PI3K inhibitor LY294002 has been shown to trap embryonic stem cells in the G1/G2 phase. Knockout of the negative regulator of PI3K pathway PTEN has been shown to be associated with increased ES proliferation [20]. It has also been reported that Delta-like 1 can inhibit differentiation of mouse embryonic cells ATDC5 into preadipocytes and osteoblasts by blocking the PI3K-AKT pathway [21]. Ke et al. found that asperosaponin, an active component of the Traditional Chinese Herbal Medicine Dipsacaceae, can activate the PI3K-AKT signaling pathway to promote differentiation of bone marrow stromal stem cells into osteoblasts in ovariectomized rats [22]. A study on the differentiation of human MSCs into osteoblasts showed that specific PI3K inhibitor LY294002 inhibited human MSCs proliferation while promoting their differentiation toward osteoblasts and mineralization. $\mathrm{Li}$ et al. reported similar findings: They found addition of specific PI3K inhibitor LY294002 to culture of condyle chondrocytes could inhibit chondrocyte 
proliferation relative to the control $(P=0.00208$, day 7$)$, and increased chondrocyte hypertrophy and type II collagenase expression $(P=0.00349)$ [23].

IFN- $\beta$ has anti-viral and immune-regulating effects, and it can block IFN- $\gamma$ synthesis, inhibit the release of cytokines harmful to oligodendrocytes, decrease $\mathrm{T}$ cell proliferation, enhance activity of regulatory $\mathrm{T}$ cells, and suppress antigen-presenting cell MHC-II expression [24]. IFN- $\beta$ is commonly used in clinical settings for multiple sclerosis and other diseases of the nervous system. Recent studies have reported on the use of interferons to induce cell differentiation. Irudayam et al. demonstrated that IFN- $\alpha$ can stimulate the STAT-JAK pathway, resulting in increased differentiation of pluripotent stem cells [25]. Diao et al. showed that type I IFN increased the number of smooth muscle cells in an in vitro study [26]. In an in vitro study, Hirsch et al. found that low concentrations of IFN- $\beta$ could reduce apoptosis of neural progenitor cells and had direct protective effect on cells in central nervous system, suggesting that it may be suitable for treatment of multiple sclerosis [27]. Matall et al. reported that type I and type II IFNs could promote division of hematopoietic stem cells, and IFN- $\gamma$ signaling enhanced myeloid differentiation of hematopoietic stem cells [28]. We previously reported that the combination of recombinant IFN- $\beta$ and TGF- $\beta$ significantly promoted differentiation of human bone marrow stromal stem cells into chondroblasts.

In the current study, we collected samples on days 4, 7, 14 , and 21 post-induction and used qRT-PCR to measure $\mathrm{PI} 3 \mathrm{~K}$ and AKT mRNA expression in the control, TGF- $\beta 3$, and TGF- $\beta 3+$ IFN- $\beta 1$ la groups. We also used Western blot to measure expression of their protein products. qPCR results showed TGF- $\beta 3$-induced PI3K and AKT mRNA expression to be higher than in the control group, and this effect was enhanced by addition of IFN- $\beta 1$ a. Likewise, TGF- $\beta 3$ induced PI3K protein expression, and this effect was amplified by addition of IFN- $\beta 1$. Regarding AKT protein expression, however, TGF- $\beta 3$ alone mainly upregulated AKT(S473) expression, while addition of IFN- $\beta 1 \mathrm{a}$ promoted AKT(T308) expression. These results suggest that TGF- $\beta 3$ and IFN- $\beta 1$ a may work in a synergistic manner in upregulating expression of key genes in the PI3K/AKT signaling pathway, which directs differentiation of hMSCs toward chondroblasts.

\section{Acknowledgments Science and Technology Planning Project}

This research was supported in part by the Natural Science Foundation of Guangdong Province (Grant No.2018A030313048) and the Science and Technology Planning Project of Shenzhen science and Technology Innovation Committee (Grant No. JCYJ20170306144035589).

\section{Reference}

1. Tsai TL, Manner PA, Li WJ. Regulation of mesenchymal stem cell chondrogenesis by glucose through protein kinase $\mathrm{C} /$ transforming growth factor signaling. Osteoarthritis and Cartilage 21 (2013) 368-376.

2. Park JS, Yang HN, Woo DG,et al. Chondrogenesis of human mesenchymal stem cells in fibrin constructs evaluated in vitro and in nude mouse and rabbit defects models. Biomaterials 32 (2011) 1495-1507.

3. Julien F., Dan B., Richard B., et al. Mesenchymal stem cell therapy in the treatment of osteoarthritis: reparative pathways, safety and efficacy - a review. BMC Musculoskeletal Disorders ,2016, 17:230-240.

4. Mamidi M.K., Das A.K., Zakaria Z. R., et al. Mesenchymal stromal cells for cartilage repair in osteoarthritis. Osteoarthritis and Cartilage. 2016,24:1307-1316.

5. [5]. Park JS, Chu JS, Tsou AD, et al. The effect of matrix stiffness on the differentiation of mesenchymal stem cells in response to TGFb. Biomaterials 2011.32(16):3921-3930.

6. Liao J, Hu N, Zhou N, et al. Sox9 potentiates BMP2-induced chondrogenic differentiation and inhibits BMP2-induced osteogenic differentiation[J]. PLo S One, 2014, 9(2) : e89025.

7. Peng Y, Li P, Zhao Z A, et al. Comparative evaluation of the wound-healing potency of recombinant bFGF and ski gene therapy in rats[J]. Growth Factors, 2016, 34(3-4): 119-127.

8. Lorenz U., Hans P. M., Lorenz M.. Insulin-like growth factor I releasing silk fibroin scaffolds induce chondrogenic differentiation of human mesenchymal stem cells. Journal of Controlled Release 2008 ,127: 12-21.

9. Lin Z, Wang J S, Lin L, et al. Effects of BMP - 2 and VEGF165 on the osteogenic differentiation of rat bone marrow - derived mesenchymal stem cells [J]. Exp Ther Med, 2014, 7(3): 625-629

10. Donovan J, Abraham D, Norman J. Platelet-derived growth factor signaling in mesenchymal cells[J]. Front Biosci,2013, 18(1) : 106-119.

11. Chan BY, Little CB. The interaction of canonical bone morphogenetic protein and Wnt-signaling pathways may play an important role in regulating cartilage degradation in osteoarthritis [J] .Arthritis Res Ther, 2012, 14(3): 119.

12. Zhang Y, Pizzute T, Pei M. A review of crosstalk between MAPK and Wnt signals and its impact on cartilage regeneration [J]. Cell Tissue Res, 2014, 358: 633-649.

13. Zhang Y, Pizzute T, Pei M. A review of crosstalk between MAPK and Wnt signals and its impact on cartilage regeneration [J]. Cell Tissue Res, 2014, 358: 633-649.

14. Oldershaw RA1, Hardingham TE. Notch signaling during chondrogenesis of human bone marrow stem cells[J]. Bone, 2010, 46(2): 286-293.

15. Pan Q, Yu Y, Chen Q, et al. Sox9, a key transcription factor of bone morphogenetic protein- 2- induced chondrogenesis, is activated through BMP pathway and a CCAAT box in the proximal promoter[J]. J Cell Physiol, 2008, 217(1): 228-241.

16. Qiaoqiao Li, Lijun Zhang, Zhili Huang, et al. Effects of recombinant human interferon $\beta 1 \mathrm{a}$ on the chondrogenic differentiation of human bone marrow mesenchymal stem cell. Chin J Biotech. 2018, 34(8): 1-9. DOI: 10.13345/j.cjb.170443

17. Jason SL,Wei Cui. Proliferation, survival and metabolism: the role of PI3K/AKT/mTOR signaling in Pluripotency and cell fate determination. Development.2016,143:3050-3060

18. Polivka Jr., J., Janku, F.. Molecular targets for cancer therapy in the PI3K/AKT/ mTOR pathway. Pharmacol. Ther. 2014,142: $164-175$.

19. Mayer, I.A., Arteaga, C.L.. The PI3K/AKT pathway as a target for cancer treatment. Annu. Rev. Med. 2016,67: 11-28.

20. Welham MJ, Storm MP, Kingham E. Phosphoinositide 3-kinases and regulation of embryonic stem cell fate. Biochemical Society transactions.2007,35(Pt2):225-228

21. Li Chen,Diyako Qanie,Abbas Jafari,et al. Delta-like 1/Fetal Antigen-1(Dlk1/FA1) is a Novel Regulator of Chondrogenic 
Cell Differentiation via Inhibition of the Akt Kinase-dependent Pathway. The Journal of Biological Chemistry.2011,286(37):32140-32149.

22. Ke K, Li Q, Yang X, et al. Asperosaponin VI promotes bone marrow stromal cell osteogenic differentiation through the PI3K/AKT signaling pathway in an osteoporosis model. Sci Rep. 2016 Oct 19; 6:35233. doi: 10.1038/srep35233.

23. Li-Li Chen,Mei Huang,Jing-Yi Tan, et al.PI3K/AKT Pathway Involvement in the Osteogenic Effects of Osteoclast Culture Supernatants on Preosteoblast Cells. Tissue Engineering: Part A 2013, doi: 10.1089/ten. tea.2012.0469

24. Joseph I. I., Deisy C.. Characterization of type I interferon pathway during hepatic differentiation of human pluripotent stem cells and hepatitis $\mathrm{C}$ virus infection. Stem Cell Research .2015,15:354-364.
25. Irudayam JI, Contreras D, Spurka L, Subramanian A,el at.Characterization of type I interferon pathway during hepatic differentiation of human pluripotent stem cells and hepatitis $\mathrm{C}$ virus infection[J]. Stem Cell Res, 2015, 15(2): 354-364.

26. Diao Y, Mohandas R, Lee P, el at.Effects of Long-Term Type I Interferon on the Arterial Wall and Smooth Muscle Progenitor Cells Differentiation[J]. Arterioscler Thromb Vasc Biol, 2016 36(2): 266-273.

27. Hirsch M, Knight J, Tobita M, el at.The effect of interferon-beta on mouse neural progenitor cell survival and differentiation $[\mathrm{J}]$. Biochem Biophys Res Commun, 2009, 388(2): 181-186

28. Matatall KA, Shen CC, Challen GA, King KY. Type II Interferon Promotes Differentiation of Myeloid-Biased Hematopoietic Stem Cells[J]. Stem Cells, 2014, 32(11): 3023-3030. 